\title{
A Critical Study of Agglomerated Multigrid Methods for Diffusion
}

\author{
Hiroaki Nishikawa, National Institute of Aerospace, Hampton, VA 23666* \\ Boris Diskin, National Institute of Aerospace, Hampton,VA $23666^{\dagger}$ \\ James L. Thomas, NASA Langley Research Center, Hampton VA 23681
}

\begin{abstract}
Agglomerated multigrid techniques used in unstructured-grid methods are studied critically for a model problem representative of laminar diffusion in the incompressible limit. The studied target-grid discretizations and discretizations used on agglomerated grids are typical of current node-centered formulations. Agglomerated multigrid convergence rates are presented using a range of two- and three-dimensional randomly perturbed unstructured grids for simple geometries with isotropic and highly stretched grids. Two agglomeration techniques are used within an overall topology-preserving agglomeration framework. The results show that multigrid with an inconsistent coarse-grid scheme using only the edge terms (also referred to in the literature as a thin-layer formulation) provides considerable speedup over single-grid methods but its convergence deteriorates on finer grids. Multigrid with a Galerkin coarse-grid discretization using piecewise-constant prolongation and a heuristic correction factor is slower and also grid-dependent. In contrast, grid-independent convergence rates are demonstrated for multigrid with consistent coarse-grid discretizations. Actual cycle results are verified using quantitative analysis methods in which parts of the cycle are replaced by their idealized counterparts.
\end{abstract}

\section{Introduction}

Multigrid techniques ${ }^{1}$ are used to accelerate convergence of current Reynolds-Averaged Navier-Stokes solvers for steady and unsteady flow solutions, especially for structured-grid applications. Mavriplis et al. ${ }^{2-5}$ pioneered agglomerated multigrid methods for large-scale unstructured-grid applications. Impressive improvements in efficiency over single-grid computations have been demonstrated. During a recent development of multigrid methods for unstructured grids, ${ }^{6}$ it was realized that some of the current approaches for coarse-grid discretization of viscous fluxes used in stateof-the-art codes have serious limitations on highly-refined grids. The purpose of this paper is to study critically the current techniques for a simple Poisson equation (representing laminar diffusion in the incompressible limit), assess their performance in grid refinement, and develop improved approaches.

The paper is organized as follows. The model diffusion equation is presented from a general finite-volume discretization (FVD) standpoint. Elements of multigrid algorithms are described, including a tabulation of target and coarse-grid discretizations. Analysis methods, in which parts of the actual multigrid cycle are replaced by their idealized counterparts, are described in the next section. The target grids and typical agglomerated grids developed within a topology-preserving framework are next shown, followed by two dimensional (2D) and three dimensional (3D) results. Results from applying analysis methods to 3D computations are also reported. The final section contains conclusions.

\section{Model Diffusion Equation and Boundary Conditions}

The FVD schemes considered are derived from the integral form of the diffusion equation,

\footnotetext{
*Research Scientist, 100 Exploration Way, hiro@nianet.org.

†Senior Research Scientist, 100 Exploration Way, bdiskin@nianet.org; Visiting Associate Professor, Mechanical and Aerospace Engineering Department, University of Virginia, Charlottesville. This research was supported by the National Institute of Aerospace under NASA Fundamental Aeronautics Program through NRA Contract NNL07AA23C and Cooperative Agreement NCC1-02043.

‡ Senior Research Scientist, Computational Aerosciences Branch, Mail Stop 128, Fellow AIAA, James.L.Thomas@nasa.gov.

This material is declared a work of the U.S. Government and is not subject to copyright protection in the United States.2006
} 


$$
\oint_{\Gamma}(\nabla U \cdot \hat{\mathbf{n}}) d \Gamma=\iint_{\Omega} f d \Omega,
$$

where $f$ is a forcing function independent of the solution $U, \Omega$ is a control volume with boundary $\Gamma, \hat{\mathbf{n}}$ is the outward unit normal vector, and $\nabla U$ is the solution gradient vector. The boundary conditions are taken as Dirichlet, i.e., specified from a known exact solution over the computational boundary. Tests are performed for simple manufactured solutions, namely collections of polynomial or sine functions. The corresponding forcing functions are found by substituting these solutions into the differential form of the diffusion equation,

$$
\Delta U=f
$$

and boundary conditions. The discretization error, $E_{d}=U-U^{h}$, is defined as the difference between the exact continuous solution, $U$, to the differential equation (2) and the exact discrete solution, $U^{h}$, of the discretized equation (1). The algebraic error is the difference between the approximate and exact discrete solutions. A scheme is considered as design-order accurate, if its discretization errors computed on a sequence of consistently-refined grids ${ }^{7,8}$ converge with the design order in the norm of interest.

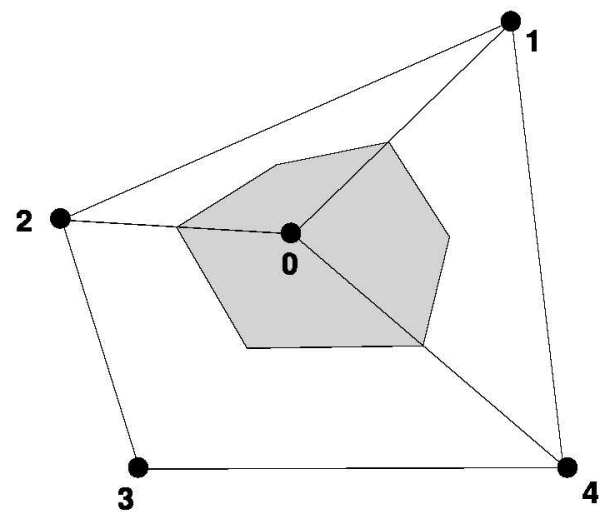

Figure 1. Illustration of a node-centered median-dual control volume (shaded) in FVD schemes. Numbers $0-4$ denote grid nodes.

The general FVD approach requires partitioning the domain into a set of non-overlapping control volumes and numerically implementing equation (1) over each control volume. Node-centered schemes define solution values at the mesh nodes. In 2D, the primal meshes are composed of triangular and quadrilateral cells; in 3D, the primal cells are tetrahedral, prismatic, or hexahedral. The median-dual partition ${ }^{9,10}$ used to generate control volumes is illustrated in Figure 1 for 2D. These non-overlapping control volumes cover the entire computational domain and compose a mesh that is dual to the primal mesh.

The control volumes of each agglomerated grid are found by summing control volumes of a finer grid. Any agglomerated grid can be defined in terms of a conservative agglomeration operator, $R_{0}$, as

$$
\Omega^{c}=R_{0} \Omega^{f},
$$

where superscripts $c$ and $f$ denote entities on coarser and finer grids, respectively. On the agglomerated grids, the control volumes become geometrically more complex than their primal counterparts and the details of the controlvolume boundaries are not retained. The directed area of a coarse-grid face separating two agglomerated control volumes, if required, is found by lumping the directed areas of the corresponding finer-grid faces and is assigned to the virtual edge connecting the centers of the agglomerated control volumes.

\section{Multigrid}

Elements of the multigrid algorithm are presented in this section. A V-cycle, ${ }^{1}$ denoted as $V\left(\nu_{1}, \nu_{2}\right)$, uses $\nu_{1}$ relaxations performed at each grid before proceeding to the coarser grid and $\nu_{2}$ relaxations after coarse-grid correction; 
the coarsest grid is solved exactly (with many relaxations). Residuals, $r$, corresponding to the integral equation (1) are restrticted to the coarse grid using $R_{0}$, as

$$
r^{c}=R_{0} r^{f} .
$$

The prolongations $P_{0}$ and $P_{1}$ are exact for piecewise-constant and linear functions, respectively. The prolongation $P_{0}$ is the transpose of $R_{0}$. The operator $P_{1}$ is constructed locally using linear interpolation from a triangle (2D) or tetrahedra (3D) defined on the coarse grid. The geometrical shape is anchored at the coarser-grid location of the agglomerate that contains the given finer control volume. Other nearby points are found using the adjacency graph. An enclosing simplex is sought that avoids prolongation with non-convex weights and, in situations where multiple geometrical shapes are found, the first one encountered is used. Where no enclosing simplex is found, the simplex with minimal non-convex weights is used. The coarse-grid solution approximation is restricted as

$$
U^{c}=\frac{R_{0}\left(U^{f} \Omega^{f}\right)}{\Omega^{c}} .
$$

The correction $\delta U$ to the finer grid is prolonged typically through $P_{1}$, as

$$
(\delta U)^{f}=P_{1}(\delta U)^{c} .
$$

\begin{tabular}{lc}
\hline \hline Target-Grid Discretizations \\
\hline Green-Gauss Avg-LSQ \\
\hline \hline
\end{tabular}

Table 1. Summary of target-grid discretizations.

The available target-grid discretizations are listed in Table 1. These schemes are representative of viscous discretizations used in Reynolds-Averaged Navier-Stokes unstructured-grid codes. The accuracy and efficiency of these discretizations have been studied in Ref. [6]; based on that study, the main target discretizations of interest is the GreenGauss scheme, which is the most widely-used viscous discretization for node-centered schemes and is equivalent to a Galerkin finite-element discretization for triangular/tetrahedral grids. For mixed elements, edge-based contributions are used to increase the $h$-ellipticity of the operator. The avg-LSQ scheme uses the average of the dual-volume LSQ gradients to determine a gradient at the face, which is augmented with the edge-based directional contribution to determine the gradient used in the flux.

A full linearization has been implemented for the Green-Gauss scheme, enabling a robust multicolor Gauss-Seidel relaxation. The avg-LSQ scheme has a comparatively larger stencil and its full linearization has not been implemented; instead relaxation of the avg-LSQ scheme relies on an approximate linearization, which consists of edge terms only. We observe good smoothing rates with this approach. The estimates for the smoothing rates obtained with quantitative analysis methods ${ }^{12}$ are shown in Section III. Note that the Green-Gauss scheme relies on an element-based data structure and hence is not considered for agglomerated grids.

\begin{tabular}{cccc}
\hline \hline \multicolumn{2}{c}{ Direct Discretization } & \multicolumn{2}{l}{ Galerkin Discretization } \\
\hline Avg-LSQ & Edge-Terms-Only & $R_{0} A^{f} P_{0}^{*}$ & $R_{0} A^{f} P_{1}$ \\
\hline \hline
\end{tabular}

Table 2. Summary of coarse-grid discretizations.

The available coarse-grid discretizations are summarized in Table 2, listing two possible direct discretizations and two possible Galerkin discretizations. The edge-terms-only discretization is often cited as a thin-layer discretization in the literature; it is a positive scheme but is not consistent (i.e., its discrete solution does not converge to the exact continuous solution with consistent grid refinement) unless the grid is orthogonal. ${ }^{7,8,11}$ An orthogonal grid would have each edge connecting solution values across a face being co-linear with the face-normal direction $\hat{\mathbf{n}}$.

The Galerkin coarse-grid operator ${ }^{1}$ is denoted by $R A P$. Because the governing equation is a second-order equation, the Galerkin construction, $R_{0} A^{f} P_{0}$, is formally inconsistent; the heuristic correction factor adopted by Mavriplis ${ }^{2}$ is used,

$$
A^{c}=R_{0} A^{f} P_{0}^{*}=\frac{1}{2} R_{0} A^{f} P_{0} .
$$


The correction factor is derived by enforcing consistency on uniformly-agglomerated hexahedral meshes. Dirichlet boundary conditions are enforced strongly. The coarse-grid operator is overwritten with the boundary condition linearization at boundary nodes.

\section{Quantitative Analysis of Unstructured Multigrid Solvers}

The quantitative analysis methods for unstructured multigrid solvers considered in this section are idealized relaxation (IR) and idealized coarse-grid (ICG) iterations introduced in Ref. [12]. The methods analyze the main complementary parts of a multigrid cycle: relaxation and coarse-grid correction. In multigrid, relaxation and coarse-grid correction are assigned to perform certain tasks: relaxation is required to smooth the algebraic error and coarse-grid correction is required to reduce smooth algebraic errors.

To apply the analysis, we first choose a desired sample fine-grid solution (zero is a natural choice for linear problems) and substitute it into the equations to generate the corresponding source and boundary data. Then we form an initial guess (for example, a random perturbation of the solution); thus, the fine-grid algebraic error is known. In the analysis, idealized iterations probe the actual two-grid cycle to identify parts limiting the overall efficiency. In these iterations, one part of the cycle is actual, and its complementary part is replaced with an idealized part. The idealized parts do not depend on the operators to be solved. They are numerical procedures acting directly on the known algebraic error to efficiently fulfill the task assigned to the corresponding part of the two-grid cycle. The results of the analysis are not single-number estimates; they are rather convergence patterns of the iterations that may either confirm or refute our expectations as to what part of the actual cycle is not efficient in carrying out the assigned task. These IR and ICG analysis methods can be regarded as a numerical extension of the Fourier analysis to problems where the classical Fourier analysis is inapplicable, in particular, to unstructured-grid solvers.

IR and ICG iterations are analysis methods that test computational efficiency of a two-grid cycle. The two-grid cycle amplification matrix, $M$, transforms the initial fine-grid algebraic error, $\mathrm{e}^{\text {old }}$, into the after-cycle error, $\mathrm{e}^{\text {new }}$,

$$
\mathbf{e}^{\text {new }}=M \mathbf{e}^{\text {old }} \text {. }
$$

The amplification matrix can be defined as

$$
M=S^{\nu_{2}} C S^{\nu_{1}} .
$$

Here, $\nu_{1}$ and $\nu_{2}$ are small nonnegative integers representing the number of pre- and post-relaxation sweeps, $S$ is the fine-grid relaxation amplification matrix, and $C$ is the amplification matrix of the coarse-grid correction.

$$
C=E-P_{0}\left(A^{c}\right)^{-1} R_{0} A^{f},
$$

where $A^{c}$ and $A^{f}$ are the coarse and fine grid operator matrices, $P_{0}$ and $R_{0}$ are the prolongation and agglomeration matrices, and $E$ is the fine-grid identity matrix.

For IR iterations, the coarse-grid correction part is actual and the relaxation is idealized. The idealized relaxation may be defined as an explicit error-averaging procedure. In this paper, we employ the IR procedure that replaces the algebraic error at each dual cell with an average of algebraic errors at edge-adjacent cells. At each relaxation step, the known exact solution, if not zero, is subtracted from the current approximation to obtain the algebraic error function. The explicit averaging procedure is applied directly to the error function. Six sweeps throughout the grid are typically performed. The exact solution is then added back. Slow convergence of IR iterations indicates insufficient coarse-grid correction.

In ICG iterations, the relaxation scheme is actual and the coarse-grid correction is idealized. Assuming that the agglomeration and prolongation operators are suitable for efficient multigrid solution, the idealized coarse-grid correction involves ideal fine and coarse operators $A^{f}$ and $A^{c}$, such that $D_{\Omega}^{c}\left(A^{c}\right)^{-1}$ is an accurate approximation to $D_{\Omega}^{f}\left(A^{f}\right)^{-1}$ for smooth error components. Here, $D_{\Omega}^{c}$ and $D_{\Omega}^{f}$ are diagonal matrices with corresponding coarse- and fine-grid volumes on the main diagonals. The best approximation is provided by the identity matrices. With this choice, the idealized coarse-grid correction becomes

$$
C^{\text {ideal }}=E-P_{0}\left(D_{\Omega}^{c}\right)^{-1} R_{0} D_{\Omega}^{f}
$$

Note that the operator $\left(D_{\Omega}^{c}\right)^{-1} R_{0} D_{\Omega}^{f}$ represents volume-weighted averaging. In ICG analysis, the ideal coarse-grid correction replaces the actual one. The idealized $C^{\text {ideal }}$ is applied directly to the known algebraic errors obtained after pre-relaxation sweep(s) of the actual relaxation. In implementation, the algebraic error is averaged to the coarse grid, changed in sign, and then prolonged to the fine grid. Slow convergence observed in the ICG iterations is a sign of poor smoothing in relaxation. 


\section{Target Grids and Agglomerations}

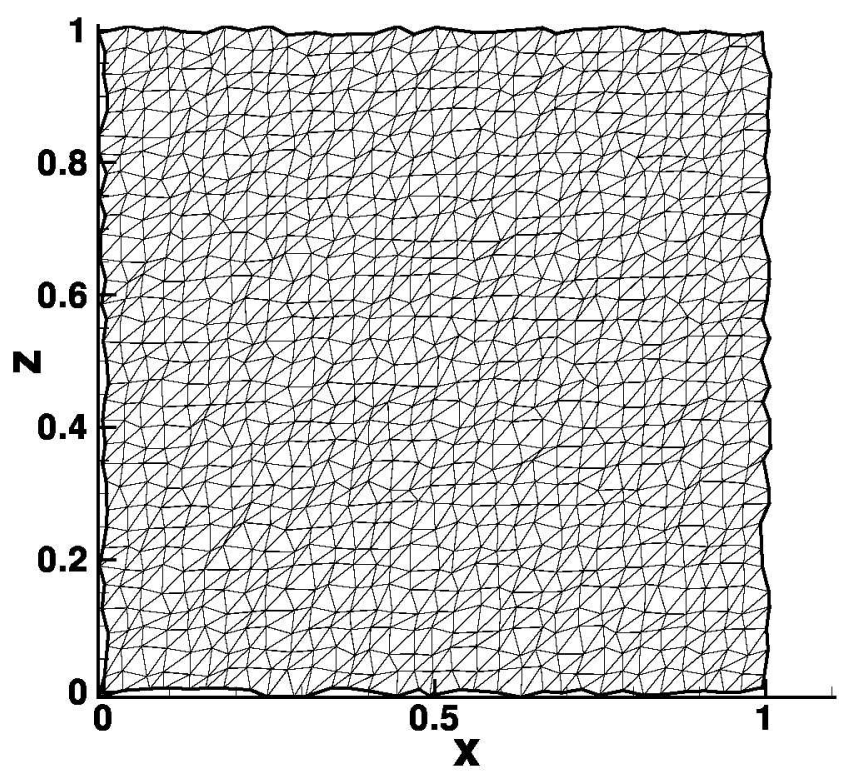

Figure 2. A typical 2D target grid.

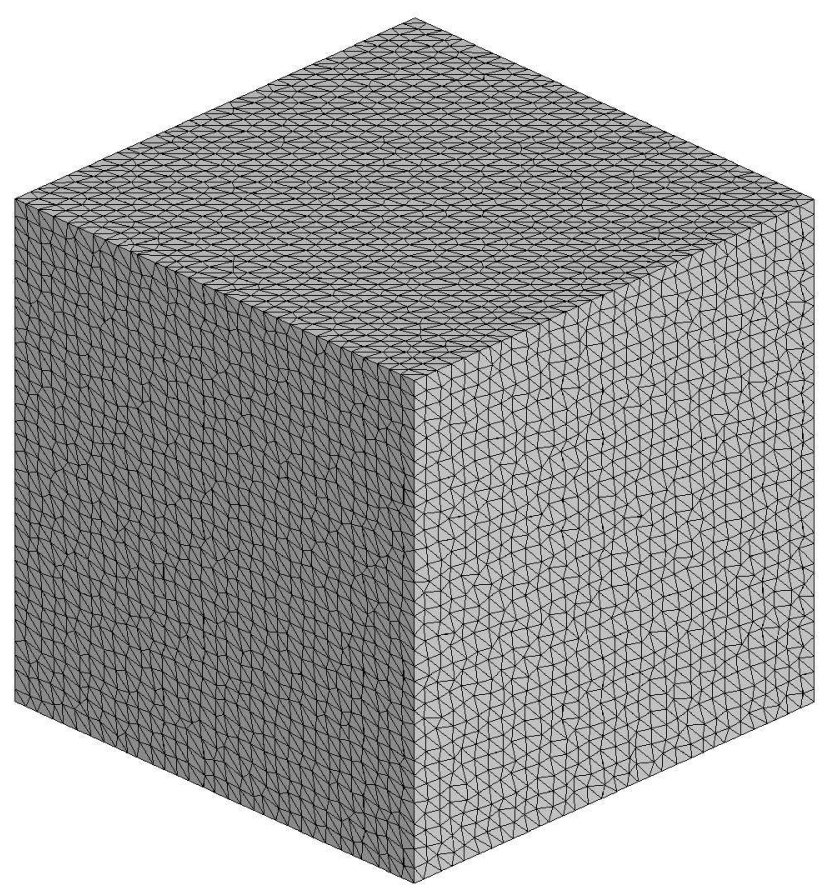

Figure 3. Orthographic view of a typical 3D target grid.

The grids considered are generated by splitting isotropic mapped Cartesian grids into triangular (2D) or tetrahedral elements (3D) and then randomly perturbing the grid points by one quarter of the local mesh size. A typical target grid is shown in Figure 2 for 2D with 33 points in each direction. An orthographic view of the boundary grids of a typical target 3D grid is shown in Figure 3, again for 33 points in each direction. 

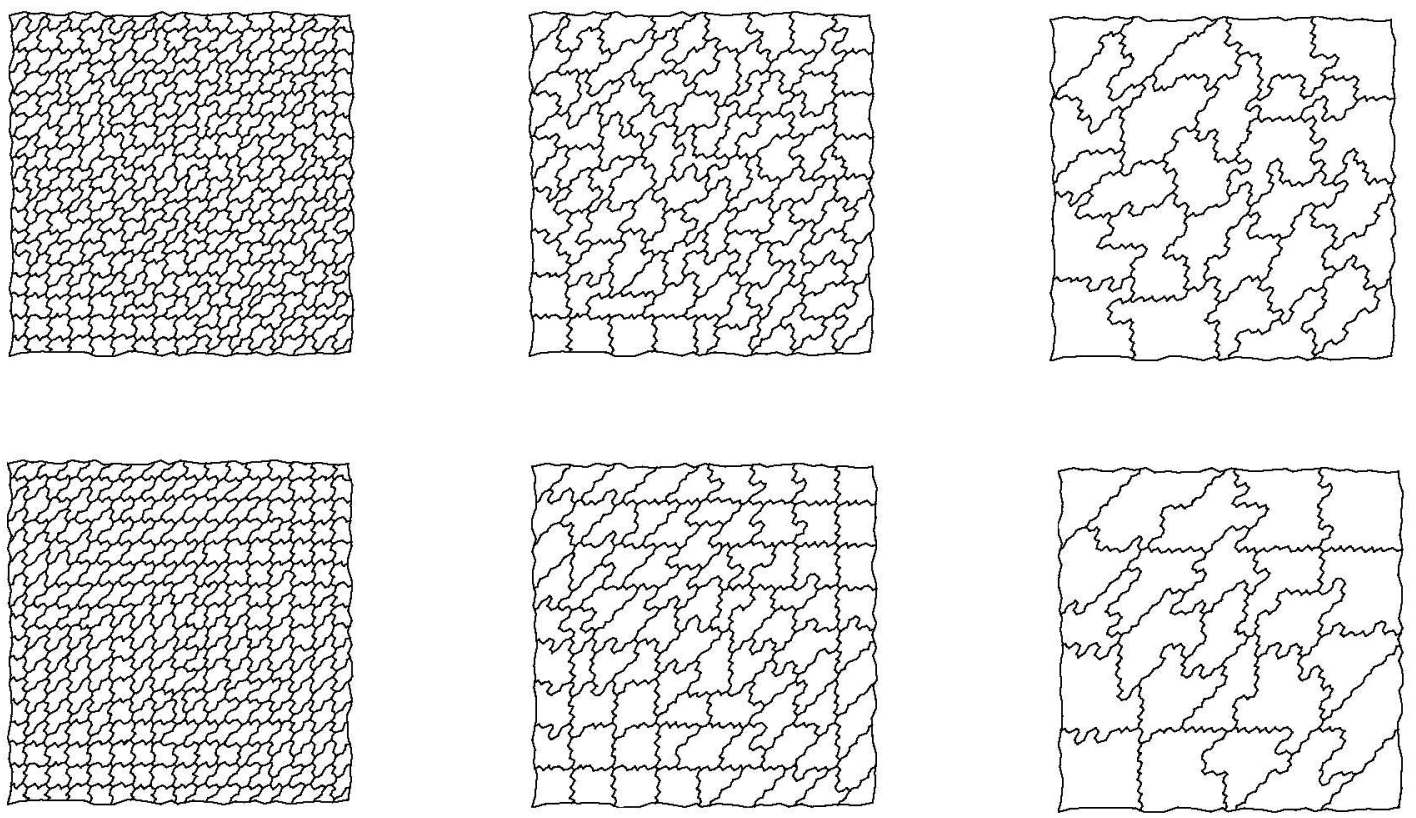

Figure 4. Control volume boundaries (non-lumped) for 2D agglomerations using scheme 1 (top row) and scheme 2 (bottom row).
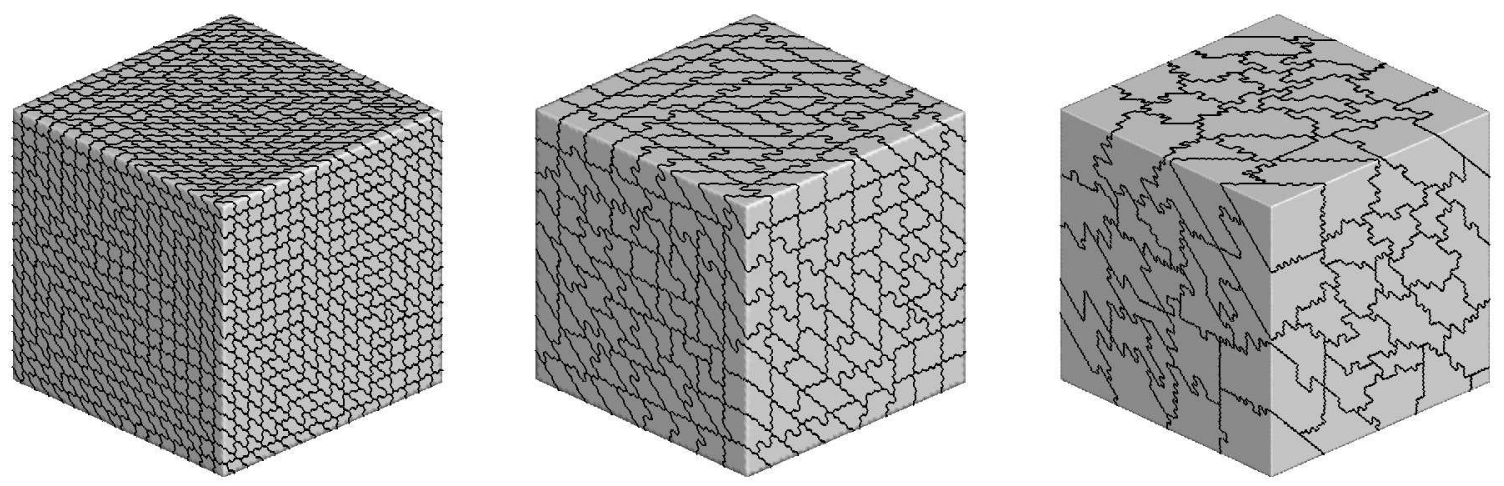

Figure 5. Control volume boundaries (non-lumped) for 3D agglomerations using scheme 2.

The grids are agglomerated within a topology-preserving framework, in which hierarchies are assigned based on connections to the computational boundaries. Corners are identified as grid points with three or more boundarycondition-type closures (or three or more boundary slope discontinuities). Ridges are identified as grid points with two boundary-condition-type closures (or two boundary slope discontinuities). Valleys are identified as grid points with a single boundary-condition-type closure and interiors are identified as grid points with no boundary closure. The agglomerations proceed hierarchically from seeds within the topologies, first corners, then ridges, then valleys, and finally interiors. Rules are enforced to maintain the boundary condition types of the finer grid within the agglomerated grid. Candidate volumes to be agglomerated are vetted against the hierarchy of the currently agglomerated volumes using the rules summarized in Table 3 . The allowed entries denote that interior volumes can be agglomerated to any existing agglomerate. The single disallowed entry enforces that two corners cannot be agglomerated. The conditional entries denote that further inspection of the connectivity of the topology must be considered before agglomeration is allowed. For example a ridge can be agglomerated into a corner if the ridge is part of the boundary condition 


\begin{tabular}{ccc}
\hline \hline Hierarchy of Agglomeration & Hierarchy of Added Volume & Agglomeration Decision \\
\hline corner & interior & allowed (corner to interior) \\
\hline corner & valley & conditional \\
\hline corner & ridge & conditional \\
\hline corner & corner & disallowed (two corners) \\
\hline ridge & interior & allowed (ridge to interior) \\
\hline ridge & valley & conditional \\
\hline ridge & ridge & conditional \\
\hline valley & interior & allowed (valley to interior) \\
\hline valley & valley & conditional \\
\hline interior & interior & allowed (interior to interior) \\
\hline \hline
\end{tabular}

Table 3. Admissible agglomerations.

specification associated with the corner. As another example, a ridge can be agglomerated into an existing ridge agglomeration if the two boundary conditions associated with each ridge are the same. Also, the prolongation operator $P_{1}$ is modified to prolong only from hierarchies equal or above the hierarchy of the prolonged point. Hierarchies on each agglomerated grid are inherited from the finer grid.

There are two agglomeration schemes, referred to as scheme 1 and 2, that have evolved historically within this development. The agglomeration scheme 1 orders the possible points within a hierarchy using the distance from the corners of the grid and closest points are taken first. Given a seed, a triad is constructed using a surrounding cloud of points, defined from the adjacency list. The first leg of the triad is defined by the seed and the nearest point. The next leg of the triad is defined by including another point from the entries in the cloud such that the leg is most orthogonal to the first leg. The third leg is found as the one most parallel to the cross-product of the first two legs. Points within the volume defined by the triads (extended to infinite length) are taken, first for the edge-adjacencies in the cloud and subsequently for the entire adjacency, to satisfy a global coarsening goal (4 volumes agglomerated for $2 \mathrm{D}$ and 8 for 3D). The agglomeration scheme 2 also starts from corners. After all corners have been agglomerated, a front list is defined by collecting nodes adjacent to the agglomerated corners. It then proceeds to agglomerate nodes in the list (while updating the list as agglomeration proceeds) in the following order: ridges, valleys, interior. A node is selected among those in the same hierarchy, that has the least number of non-agglomerated neighbors to reduce the occurrences of agglomerations with small numbers of volumes. For a given seed, it collects all neighbors and agglomerates them up to a specified maximum number, e.g., 8 in 3D. The agglomeration continues until the front list becomes empty. For either agglomeration scheme, agglomerations containing only a few volumes are combined with other agglomerations, as is typical of the methods used in the literature.

For highly stretched meshes, the agglomerations are first constructed along the boundary of the grid (corners, ridges, and valleys) and then the cells are agglomerated from the boundary to the ends of the implicit lines associated with the stretched grid. The boundary agglomerate is merged with the volumes corresponding to the next point in the line. The agglomeration continues to the end of the shortest line in the boundary agglomerate, forming an agglomeration from the volumes associated with the next two entries in the line. After agglomeration of lines, the algorithm uses the point agglomeration method for the rest of the domain.

Figure 4 shows three agglomerated grids generated from the primal grid in Figure 2 using schemes 1 and 2 . Figure 5 shows three agglomerated grids generated from the primal grid in Figure 3 using scheme 2 . The agglomerations are representative of those in the literature.

\section{Two Dimensional Results}

A summary of multigrid convergence rates are compiled in Tables 4 and 5 for the two agglomeration schemes, respectively, for a $\mathrm{V}(2,1)$ multigrid cycle with various coarse-grid operators. Multigrid cycles employ as many levels

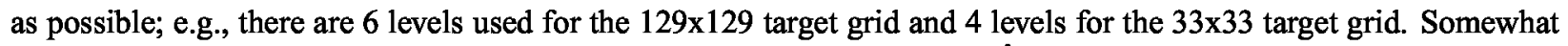
surprisingly, with the Galerkin coarse-grid operator constructed via $R_{0} A^{f} P_{1}$, the multigrid algorithm is divergent. The reason, confirmed by analysis, is that the coarse-grid operator, although accurate, loses $h$-ellipticity. ${ }^{1}$ This loss of $h$-ellipticity for the Galerkin operator with simplex-based $P_{1}$-prolongation has been observed even with quadrilateral 


\begin{tabular}{ccccc}
\hline \hline Fine Grid & \multicolumn{2}{c}{ Direct Discretization } & \multicolumn{2}{c}{ Galerkin Discretization } \\
\hline & Avg-LSQ & Edge-Terms-Only & $R_{0} A^{f} P_{0}^{*}$ & $R_{0} A^{f} P_{1}$ \\
\hline $33 \times 33$ & 0.15 & 0.20 & 0.51 & divergent \\
\hline $65 \times 65$ & 0.22 & 0.26 & 0.53 & divergent \\
\hline $129 \times 129$ & 0.21 & 0.32 & 0.60 & divergent \\
\hline \hline
\end{tabular}

Table 4. Summary of multi-level asymptotic convergence rates per V(2,1) multigrid cycle with agglomeration scheme 1 for various coarsegrid operators.

\begin{tabular}{ccccc}
\hline \hline Fine Grid & \multicolumn{2}{c}{ Direct Discretization } & \multicolumn{2}{c}{ Galerkin Discretization } \\
\hline & Avg-LSQ & Edge-Terms-Only & $R_{0} A^{f} P_{0}^{*}$ & $R_{0} A^{f} P_{1}$ \\
\hline $33 \times 33$ & 0.17 & 0.29 & 0.49 & divergent \\
\hline $65 \times 65$ & 0.19 & 0.44 & 0.58 & divergent \\
\hline $129 \times 129$ & 0.18 & 0.54 & 0.68 & divergent \\
\hline \hline
\end{tabular}

Table 5. Summary of multi-level asymptotic convergence rates per $V(2,1)$ multigrid cycle with agglomeration scheme 2 for various coarsegrid operators.

grids, for which bilinear prolongation is known to result in $h$-elliptic coarse-grid operators.

With the Galerkin coarse-grid operator constructed via $R_{0} A^{f} P_{0}^{*}$, the multigrid algorithm is stable. However, the convergence rates degrade on finer grids with either agglomeration scheme. With the coarse-grid operator using only the edge terms, the convergence per cycle is better, but again shows a deterioration on finer grids. The deterioration is noticeably worse with the agglomeration scheme 2 , although it is hard to judge the reason from visual inspection of the agglomerated grids. With the avg-LSQ scheme, the convergence per cycle is 0.22 or better and grid-independent.

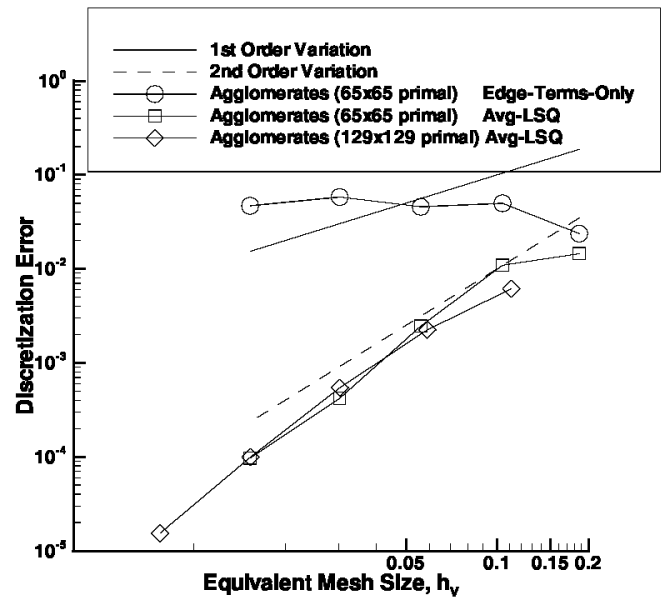

Figure 6. Spatial convergence of discretization error for agglomerate families.

The spatial convergence of discretization error for agglomerate families with the avg-LSQ target-grid discretization is shown in Figure 6. Results with the edge-terms-only (thin-layer) discretization are also shown for reference. Each agglomerate family is composed of a single primal grid and agglomerated grids generated recursively; a particular agglomerate family is denoted by the density of the primal mesh in parentheses. The $L_{1}$ norm of the discretization error is shown versus an equivalent mesh size, taken as the $L_{1}$ norm of a local characteristic distance, i.e., $h_{V}=\left\|\Omega^{1 / d}\right\|$, where $d$ is the number of spatial dimensions. The edge-terms-only discretization shows no order property, as expected, but the avg-LSQ scheme shows a second-order convergence of discretization error. This result is believed to be the first to show order properties for diffusion on a family of agglomerated meshes.

For the finer agglomerate family, multigrid convergence is shown in Figure 7 using the avg-LSQ discretization on 


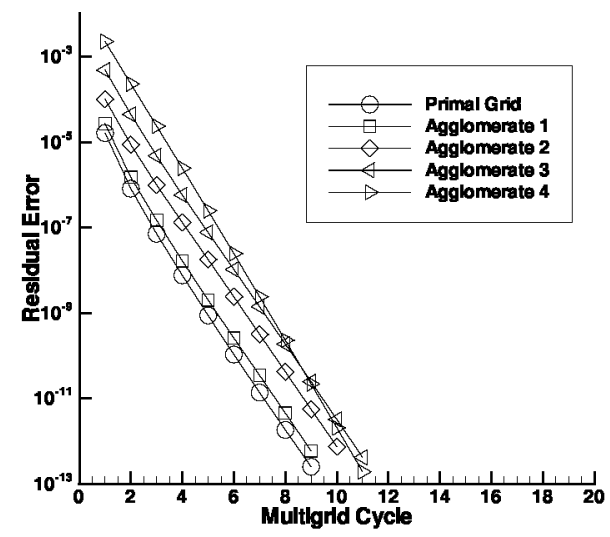

Figure 7. Multigrid convergence for agglomerate family composed of the $129 \times 129$ primal mesh and its coarsened agglomerates.

all grids. Multilevel V(2,2) cycles are used with 2 levels on the coarsest agglomerate and 6 levels on the primal mesh. The initial conditions are taken as the exact solution with a randomly perturbed error on each grid. Grid-independent convergence is shown with approximately an order of magnitude reduction in residual per cycle.

\section{Three Dimensional Results}

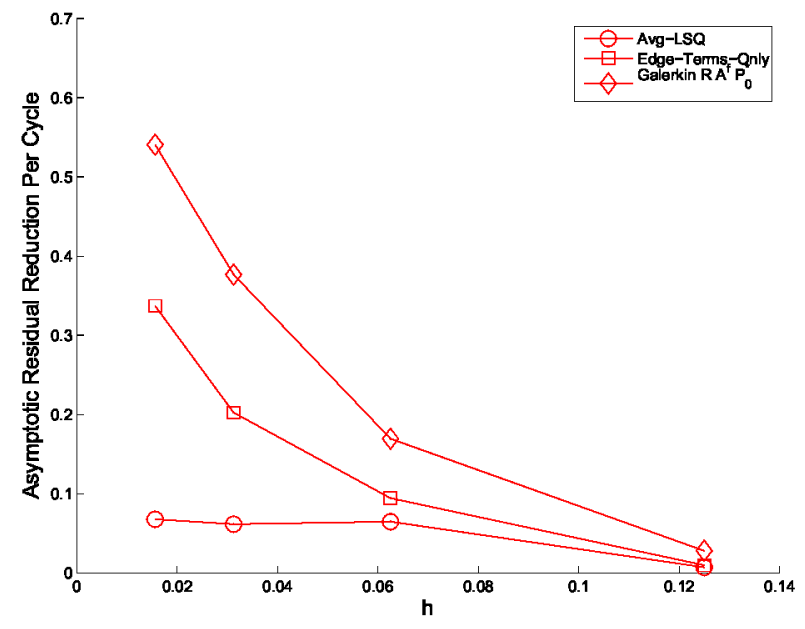

(a) 2-level.

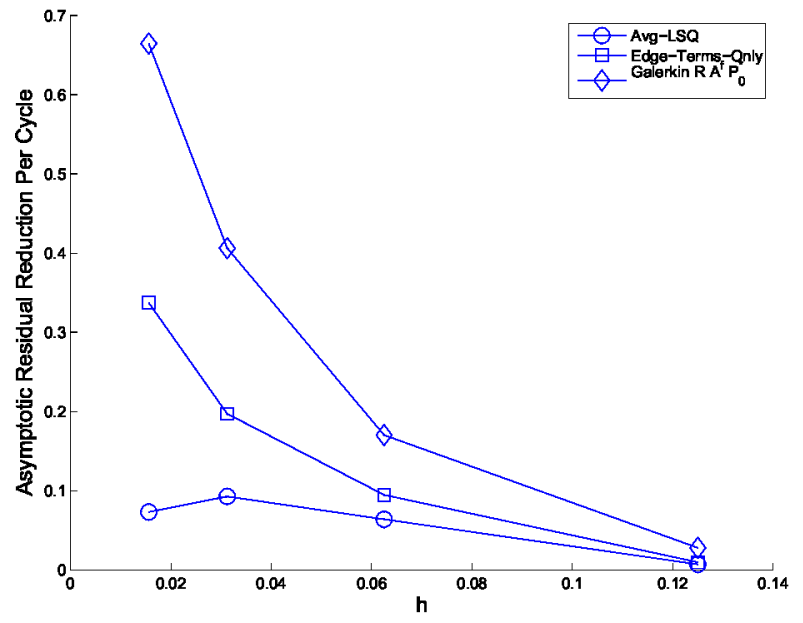

(b) Multi-level.

Figure 8. Asymptotic convergence rate per cycle for V(3,3) multigrid 2-level cycle with agglomeration scheme 2 for various coarse-grid operators.

Multigrid asymptotic convergence rates per cycle are shown in Figure 8 with various coarse-grid operators for a range of grids $(9 \times 9 \times 9$ to $65 \times 65 \times 65)$. Results were obtained with two- and multiple-level V( 3,3$)$ multigrid cycles. The mesh size $h$ corresponds to $1 /\left(N^{1 / 3}-1\right)$ where $N$ is the total number of nodes. Agglomerated grids are generated with scheme 2 . We do not show results with the Galerkin coarse-grid operator constructed via $R_{0} A^{f} P_{1}$ because the algorithm is again divergent.

The 3D results are consistent with the $2 \mathrm{D}$ results. With the Galerkin coarse-grid operator constructed via $R_{0} A^{f} P_{0}^{*}$, the multigrid algorithm is stable, but the convergence degrades on finer grids. With agglomerated grids using the edge-terms-only scheme, the convergence per cycle is better but again shows a deterioration on finer grids. With ag- 
glomerated grids using the avg-LSQ scheme, the convergence per cycle is practically grid-independent; the asymptotic convergence per cycle is similar to that in $2 \mathrm{D}$.

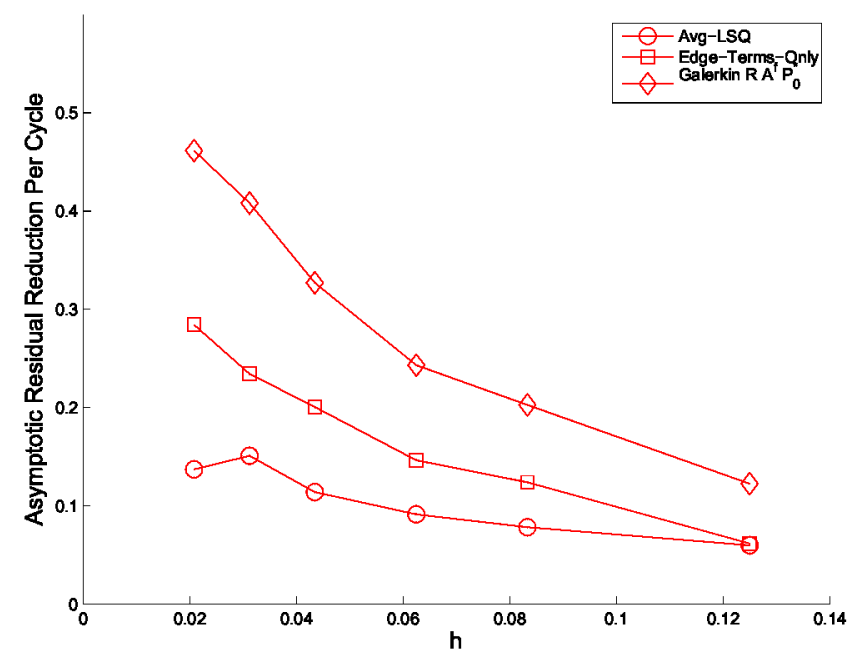

Figure 9. Asymptotic convergence rate per V(3,3) multigrid 2-level cycle with agglomeration scheme 2 and a line agglomeration/relaxation in the stretched region.

The same multigrid $V(3,3)$ was tested with a line-agglomeration/relaxation for stretched grids typical in high-

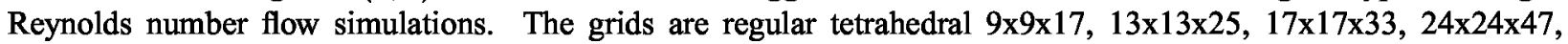
$33 \times 33 \times 65,49 \times 49 \times 97$ grids with exponential stretching applied in the $z$-direction. The stretching was applied only in the lower half region; the upper half is left isotropic. A typical grid is shown in Figure 10. We applied a lineagglomeration and a line-relaxation to the stretched region in order to effectively remove high-frequency errors on fine grids. The results are shown in Figure 9. Again, the edge-terms-only scheme and the Galerkin operator scheme show a deterioration on finer grids while the avg-LSQ scheme gives practically grid-independent results.

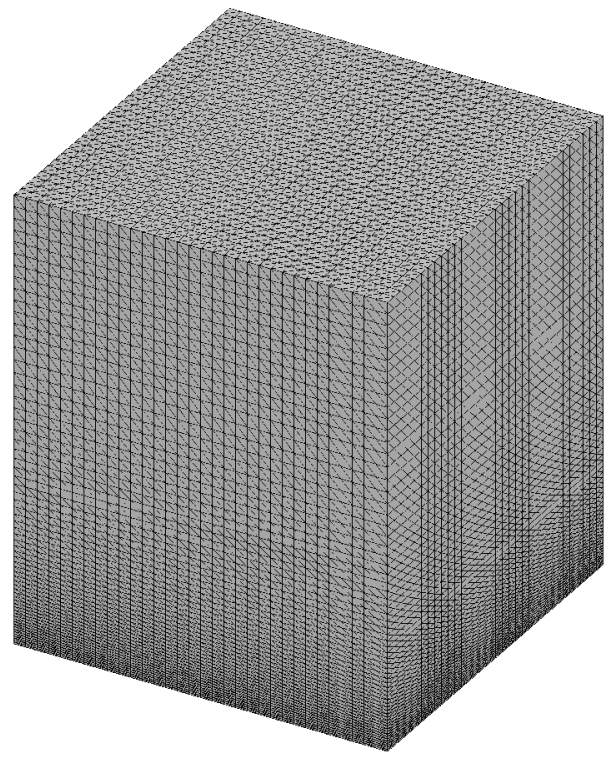

Figure 10. 33x33x65 Stretched grid with the maximum aspect ratio of 6.25 .

The IR and ICG analysis methods have been applied within a two-grid multigrid cycle on perturbed isotropic tetrahedral grids to evaluate relaxation smoothing and efficiency of coarse-grid correction. Fine-grid point relaxation

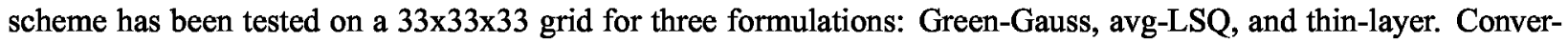
gence rates observed in ICG iterations and collected in Table 6 show that the tested relaxation is an efficient error smoother for all three schemes; the high-frequency error reduction is better than 0.55 , which is an excellent smoothing 
factor.

\begin{tabular}{ccc}
\hline \hline Green-Gauss & Avg-LSQ & Edge-Terms-Only \\
\hline 0.545 & 0.470 & 0.358 \\
\hline \hline
\end{tabular}

Table 6. Summary of smoothing rates of three relaxation schemes obtained from ICG-V(1,0) on a 33x33x33 perturbed isotropic tetrahedral grid.

IR iterations have been performed to analyze the quality of coarse-grid correction with two different coarse-grid schemes: avg-LSQ and thin-layer approximation. The results are shown in Table 7. To provide a robust gridindependent convergence rates in a multigrid cycle with many grids, the coarse-grid correction is expected to reduce smooth errors by an order of magnitude. Convergence rates observed in IR iterations with six explicit error averaging sweeps show that the coarse-grid correction is adequate for the avg-LSQ scheme. The rates observed for the edgeterms-only scheme are slow and further deteriorate on grids with high skewing. Both schemes appear insensitive to the prolongation order, demonstrating almost identical convergence rates for either $P_{0}$ or $P_{1}$ prolongation operator.

\begin{tabular}{ccc}
\hline \hline Coarse Grid & Avg-LSQ & Edge-Terms-Only \\
\hline$P_{0}$ prolongation & 0.124 & 0.303 \\
\hline$P_{1}$ prolongation & 0.125 & 0.303 \\
\hline \hline
\end{tabular}

Table 7. Summary of convergence rates for two coarse grid correction schemes obtained from IR-V(3,3) on a $33 \times 33 \times 33$ perturbed isotropic tetrahedral grid.

\section{Concluding Remarks}

Agglomerated multigrid techniques used in unstructured-grid methods have been critically studied for a model problem representative of laminar diffusion in the incompressible limit. The studied target-grid discretizations and discretizations used on agglomerated grids are typical node-centered formulations. Agglomerated multigrid convergence rates are compiled using a range of two- and three-dimensional randomly perturbed unstructured grids for simple geometries, including isotropic and highly-stretched grids. Two agglomeration techniques are used within an overall topology-preserving agglomeration framework. The results show that multigrid with an inconsistent coarse-grid scheme using only the edge terms (also referred to in the literature as a thin-layer formulation) provides considerable speedup over single-grid methods but its convergence deteriorates on finer grids. Multigrid with a formally inconsistent Galerkin coarse-grid discretization using piecewise-constant prolongation and a heuristic correction is slower and also grid-dependent. A consistent Galerkin coarse-grid construction using simplex prolongation was found to be unstable because the discretization lacked h-ellipticity. Grid-independent convergence rates are demonstrated for multigrid with consistent coarse-grid discretizations. The results from the actual cycle are verified using discrete analysis methods in which parts of the cycle are replaced by their idealized counterparts.

\section{Acknowledgments}

The three-dimensional results presented were computed within the FUN3D suite of codes at NASA Langley Research Center (http://fun3d.larc.nasa.gov/). The contributions of E. J. Nielsen, J. A. White, and R.T. Biedron of NASA in the implementation within FUN3D are gratefully acknowledged.

\section{References}

\footnotetext{
${ }^{1}$ Trottenberg U., Oosterlee, C. W. and Schüler, A., Multigrid, Academic Press, London, 2000.

${ }^{2}$ Mavriplis, D. J., "Multigrid Techniques for Unstructured Meshes," VKI Lecture Series VKI-LS 1995-02, Von Karman Institute for Fluid Dynamics, Rhode-Saint-Genese, Belgium 1995.

${ }^{3}$ Mavriplis, D. J., "Unstructured Grid Techniques," Annual Review of Fluid Mechanics, Vol. 29, 1997, pp. 473-514.

${ }^{4}$ Mavriplis, D. J., and Pirzadeh, S, "Large-Scale Parallel Unstructured Mesh Computations for 3D High-Lift Analysis," Journal of Aircraft, Vol. 36, No. 6, 1999, pp. 987-998.
} 
${ }^{5}$ Mavriplis, D. J., "An Assessment of Linear versus Non-Linear Multigrid Methods for Unstructured Mesh Solvers," Journal of Computational Physics, Vol. 175, 2002, pp. 302-325.

${ }^{6}$ Diskin, B., Thomas, J. L., Nielsen, E. N., and White, J. A., "Comparison of Node-Centered and Cell-Centered Unstructured Finite-Volume Discretizations Part I Viscous Fluxes," AIAA Paper 2009-597, January 2009.

${ }^{7}$ Diskin, B. and Thomas, J. L., “Accuracy Analysis for Mixed-Element Finite-Volume Discretization Schemes," NIA Technical Report 2007-8, August 2007.

${ }^{8}$ Thomas, J. L., Diskin, B., and Rumsey, C. L., “Towards Verification of Unstructured Grid Methods,” AIAA Journal, Vol. 46, No. 12, December 2008, pp. 3070-3079 (also AIAA Paper 2008-0666). 1991.

${ }^{9}$ Barth, T. J., "Numerical Aspects of Computing High-Reynolds Number Flow on Unstructured Meshes," AIAA Paper 91-0721, January

${ }^{10}$ Haselbacher, A. C., A Grid-Transparent Numerical Method for Compressible Viscous Flow on Mixed Unstructured Meshes, PhD thesis, Loughborough University, 1999.

${ }^{11}$ Svärd, M. and Nordström, J., "An Accuracy Evaluation of Unstructured Node-centered Finite-volume Methods," Applied Numerical Mathematics, Vol. 58, No. 8, 2008, pp. 1142-1158; also available as NIA Report 2005-04, NASA CR-2006-214293, April 2006.

${ }^{12}$ Diskin, B., Thomas, J. L., and Mineck, R., “On Quantitative Analysis Methods for Multigrid Solutions,” SIAM J. Sci. Comput., Vol. 27, No. 1, 2005, pp. 108-129. 